\title{
Medical Image of the week: Chronic Pulmonary Histoplasmosis
}

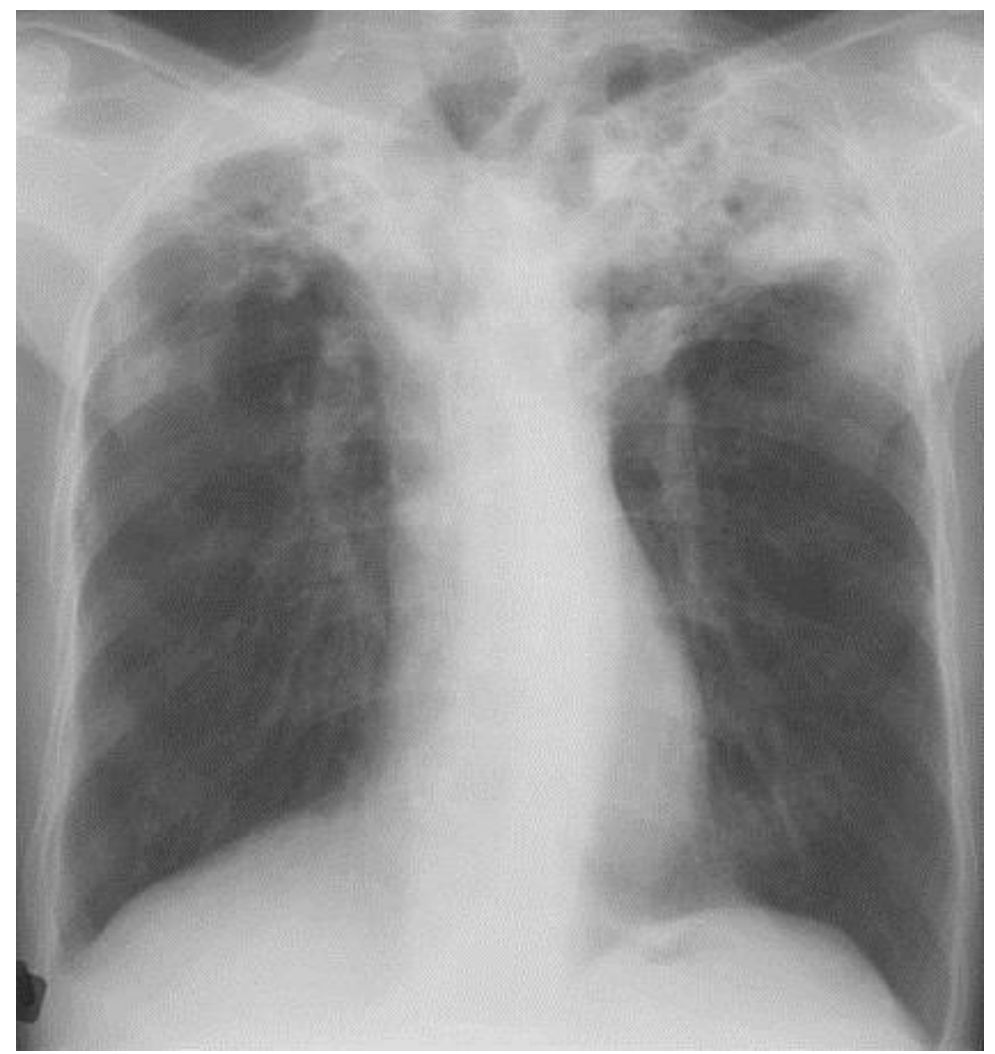

Figure 1. Chest radiography showing upper lobe fibrosis and cavitation secondary to chronic histoplasmosis.

Histoplasmosis is endemic to the Midwest US and commonly causes an acute infection that presents as a subacute pneumonia. In patients with underlying lung disease, particularly COPD, a subacute pneumonia can evolve into chronic pulmonary histoplasmosis and is characterized by persistent or recurrent pulmonary symptoms, progressive lung infiltrates, fibrosis, and cavitation. Upper lobe infiltrates and cavities are characteristic, resembling the findings in tuberculosis (Figure 1). Progression is manifested by cavity enlargement, increased fibrosis and bronchopleural fistulae. Misdiagnosis delays therapy and can be catastrophic. Histoplasmosis titers and sputum cultures are useful tests. Bronchoscopy with bronchoalveolar lavage and transbronchial biopsy for culture may be needed when diagnosis remains elusive.

Kenneth S. Knox, MD ${ }^{1}$ and Veronica A. Arteaga, $\mathrm{MD}^{2}$

${ }^{1}$ Professor of Medicine

University of Arizona College of Medicine- Phoenix

Phoenix, AZ USA

${ }^{2}$ Associate Professor of Medicine

Medical Imaging

University of Arizona College of Medicine- Tucson

Tucson, AZ USA 